\title{
Arterial Stiffness in Patients with Deep and Lobar Intracerebral Hemorrhage
}

\author{
Maurizio Acampa, Francesca Guideri, Ilaria Di Donato, Rossana Tassi, Giovanna Marotta, \\ Giuseppe Lo Giudice, Paolo D’Andrea, Giuseppe Martini \\ Stroke Unit, Department of Neurological and Sensorineural Sciences, Azienda Ospedaliera Universitaria Senese, "Santa Maria alle Scotte” General \\ Hospital, Siena, Italy
}

Background and Purpose Intracerebral hemorrhage (ICH) accounts for approximately 10\% of stroke cases. Hypertension may play a role in the pathogenesis of ICH that occurs in the basal ganglia, thalamus, pons, and cerebellum, but not in that of lobar ICH. Hypertension contributes to decreased elasticity of arteries, thereby increasing the likelihood of rupture in response to acute elevation in intravascular pressure. This study aimed to evaluate arterial stiffness (using the arterial stiffness index [ASI]) in patients with deep (putaminal and thalamic) ICH in comparison with patients with lobar ICH.

Methods We enrolled 64 patients (mean \pm SD age: $69.3 \pm 10.7$ years; 47 men and 17 women) among 73 who referred consecutively to our department for intraparenchymal hemorrhage and underwent brain computed tomography (CT) and cerebral angio-CT. In all the subjects, 24-hour heart rates and blood pressures were monitored. The linear regression slope of diastolic on systolic blood pressure was assumed as a global measure of arterial compliance, and its complement (1 minus the slope), ASI, has been considered as a measure of arterial stiffness.

Results In the patients with deep ICH, ASI was significantly higher than in the patients with lobar ICH ( $0.64 \pm 0.19$ vs. $0.53 \pm 0.17, P=0.04)$.

Conclusions Our results suggest that in deep ICH, arterial stiffening represents a possible pathogenetic factor that modifies arterial wall properties and contributes to vascular rupture in response to intravascular pressure acute elevation. Therapeutic strategies that reduce arterial stiffness may potentially lower the incidence of deep hemorrhagic stroke.

Keywords Hemorrhagic stroke; Intracerebral hemorrhage; Deep cerebral hemorrhage; Lobar cerebral hemorrhage; Arterial stiffness; Arterial hypertension

\author{
Correspondence: Maurizio Acampa \\ UOC Stroke Unit, Policlinico "S. Maria \\ alle Scotte", viale Bracci, n.1, 53100 \\ Siena, Italy \\ Tel: $+39-0-577585476$ \\ Fax: +39-0-577585307 \\ E-mail: M.Acampa@ao-siena.toscana.it \\ Received: July 1, 2014 \\ Revised: August 26, 2014 \\ Accepted: September 7, 2014
}

The authors have no financial conflicts of interest.

\section{Introduction}

Intracerebral hemorrhage (ICH) accounts for 10\%-15\% of all stroke cases ${ }^{1}$ and tends to occur earlier in life, with a higher risk of fatality, than cerebral infarction. ${ }^{2} \mathrm{ICH}$ can be broadly divided into two categories as follows: a) deep hemorrhage, which is classically associated with hypertension, and b) lobar hemor- rhage, which in many cases is suspected to result from cerebral amyloid angiopathy. ${ }^{3,4} \mathrm{ICH}$ attributed to hypertension characteristically occurs in the basal ganglia, thalamus, pons, and cerebellum; these deep regions of the brain are vulnerable to lacunar infarctions because they are served by small end arteries that branch from larger vessels. ${ }^{5}$ Indeed, the same pathology may underlie hypertensive ICH and lacunar disease; in fact, both 
types of lesions may be found simultaneously in the same patient. ${ }^{5} \mathrm{~A}$ broad consensus suggests that hypertension is the most important risk factor of $\mathrm{ICH}$, and several studies have suggested an association between vascular stiffening and hypertension. ${ }^{6}$ Arterial stiffness predicts the incidence of cardiovascular complications over and beyond traditional risk factors, ${ }^{7}$ representing an independent risk factor of symptomatic stroke. Arterial stiffening seemed to be associated with the presence of lacunar infarcts. ${ }^{8}$ A recent study ${ }^{9}$ showed higher arterial stiffness index (ASI) values in patients with acute ischemic stroke, particularly those with the lacunar subtype. Arterial stiffening represents the result of many disease states such as hypertension, diabetes, atherosclerosis, hypercholesterolemia, and chronic renal failure. ${ }^{10}$

The aim of our study was to evaluate arterial stiffening (measured using the ASI) and metabolic parameters (high-sensitivity C-reactive protein level, $\mathrm{HbAlc}$ percentage, serum total-cholesterol level, and low-density lipoprotein [LDL] and high-density lipoprotein [HDL] cholesterol levels) in patients with deep (putaminal and thalamic) ICH in comparison with patients with lobar ICH and, eventually, to suggest a possible role for such parameters in the pathogenesis of deep ICH.

\section{Methods}

Seventy-three patients, who were referred consecutively to our department for primary $\mathrm{ICH}$, underwent brain computed tomography (CT) and cerebral angio-CT. The presence of arteriovenous malformation, aneurysm, neoplasia, cavernous angioma, or coagulopathy were exclusion criteria. Sixty-four patients were enrolled in the study (Table 1), whereas 9 patients (mean \pm SD age, $70 \pm 11$ years; 5 men and 4 women) with lobar hemorrhage were excluded from this study because of secondary causes of ICH (arteriovenous malformations in 2 patients, cavernous angioma in 1 patient, arterial aneurysms in 2 patients, and cerebral venous thrombosis in 4 patients).

A 24-hour heart rate (HR) and blood pressure (BP) monitoring was conducted for all the subjects. In all the patients, highsensitivity C-reactive protein (hsCRP) level, $\mathrm{HbAlc}$ percentage, serum total-cholesterol level, and LDL- and HDL-cholesterol levels were measured. Informed consent was obtained from each patient before participating in the study. The study protocol conforms to the ethical guidelines of the 1975 Declaration of Helsinki.

\section{Arterial stiffness index}

$\mathrm{BP}$ monitoring was performed using validated oscillometric devices (Bedside Monitor Life Scope I BSM-2303K, International Division, Nihon Kohden Corp., Tokyo, Japan), during
Table 1. Hemodynamic and metabolic parameters in the patients with deep and lobar intracerebral hemorrhage

\begin{tabular}{lccc}
\hline & Deep ICH $(\mathrm{n}=45)$ & Lobar ICH $(\mathrm{n}=19)$ & $P$ \\
\hline Age (years) & $67.8 \pm 10.8$ & $72.8 \pm 9.8$ & 0.10 \\
& $(70[61-75])$ & $(75[68-79])$ & \\
Sex (female:male) & $10: 35$ & $8: 11$ & 0.13 \\
SBP (mmHg) & $141 \pm 16$ & $138 \pm 12$ & 0.43 \\
DBP (mmHg) & $75 \pm 11$ & $73 \pm 10$ & 0.45 \\
MBP (mmHg) & $96 \pm 11$ & $95 \pm 10$ & 0.95 \\
PP (mmHg) & $65 \pm 15$ & $65 \pm 11$ & 0.96 \\
HR (beats/min) & $71 \pm 12$ & $74 \pm 12$ & 0.51 \\
Total cholesterol (mg/dL) & $193 \pm 42$ & $187 \pm 38$ & 0.62 \\
HDL-cholesterol (mg/dL) & $50 \pm 15$ & $56 \pm 19$ & 0.32 \\
LDL-cholesterol (mg/dL) & $119 \pm 34$ & $113 \pm 33$ & 0.48 \\
HbA1c (\%) & $5.9 \pm 0.4$ & $5.7 \pm 0.6$ & 0.24 \\
RCP (mg/dL) & $1.38 \pm 2.0$ & $1.80 \pm 2.8$ & 0.37 \\
& $(0.6[0.29-1.25])$ & $(0.93[0.35-2.17])$ & \\
ASI & $0.64 \pm 0.19$ & $0.53 \pm 0.17$ & 0.04 \\
& $(0.68[0.49-0.77])$ & $(0.59[0.51-0.63])$ & \\
Hypertension & $39 / 45$ & $15 / 19$ & 0.46 \\
Diabetes & $16 / 45$ & $4 / 19$ & 0.37 \\
Smoking & $25 / 45$ & $7 / 19$ & 0.27 \\
\hline
\end{tabular}

The data are expressed as mean \pm SD. Those in the brackets are medians and interquartile ranges.

SBP, systolic blood pressure; DBP, diastolic blood pressure; MBP, mean blood pressure; PP, pulse pressure; $H R$, heart rate; $\mathrm{RCP}$, reactive $\mathrm{C}$ protein.

the admission period and at 24 hours from stroke onset. For 24 h, BP was recorded every 30 minutes, between $0600 \mathrm{~h}$ to midnight, and hourly, from midnight to $0600 \mathrm{~h}$. The 24-h-monitored parameter values were finally edited. For each subject, at least $80 \%$ of valid BP and HR measurements had to be obtained for inclusion in the study.

Repeated measurements of systolic BP (SBP) and diastolic BP (DBP) are well known to display similar patterns over time, with a linear correlation. However, in subjects with arterial stiffening, changes in DBP are accompanied by greater changes in SBP, reflecting a loss of arterial capacitance. This phenomenological relationship can be analyzed by plotting the individual SBP and DBP measurements that were obtained through the 24-h noninvasive monitoring (Figure 1). The linear regression slope of DBP on SBP was obtained and assumed as a global measure of arterial compliance, and its complement (1 minus the slope), ASI, has been considered as a measure of arterial stiffness. ${ }^{11,12}$ In the patients with a stiffer arterial tree, the regression slope tends to be 0 and ASI tends to be 1 .

\section{Statistical analysis}

Statistical analysis was performed using the GraphPad Instat computer software (version 3.06 for Windows, GraphPad Software Inc., La Jolla, CA, USA). All results were presented as mean \pm SD values. Data were first analyzed using the Kolmogorov- 

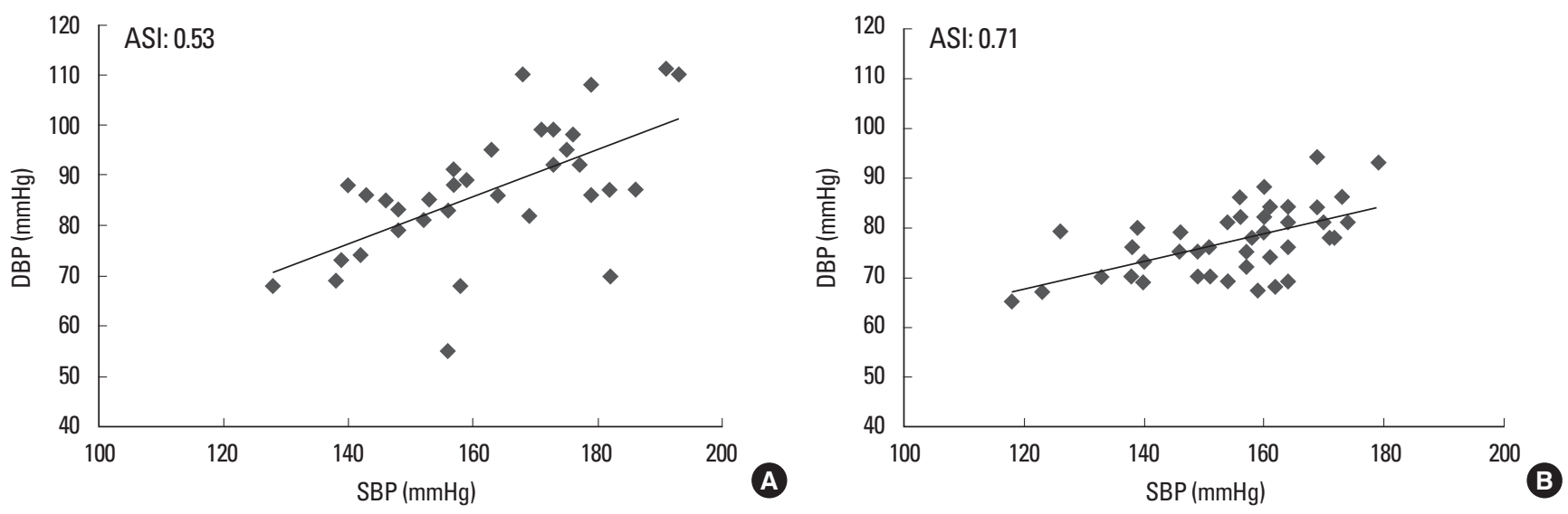

Figure 1. The linear regression slope of DBP on SBP and ASI in a patient with lobar ( $\mathrm{A})$ and deep cerebral hemorrhages $(\mathrm{B})$. The stiffer the arterial tree, the closer the regression slope and ASI to 0 and 1, respectively.

Smirnov test for data distribution normality. Statistical analysis included comparisons of ASI, age, and hsCRP level in the patients with deep and lobar ICHs, using the nonparametric MannWhitney test for unpaired data. Unpaired $t$ test was performed to compare cholesterol level, HbAlc percentage, SBP, DBP, mean $\mathrm{BP}(\mathrm{MBP})$, pulse pressure (PP), $\mathrm{HR}$ in patients with deep and lobar ICHs. Data obtained from the comparison of the numbers of hypertensive and diabetic patients in the two groups were evaluated using the Fisher exact test.

\section{Results}

In our study, we enrolled 64 patients with $\mathrm{ICH}($ mean \pm SD age: $69.3 \pm 10.7$ years; 47 men and 17 women). Deep ICH was present in 45 patients; lobar ICH, in 19 patients (Table 1). No differences were found between the patients with deep and lobar ICH with regard to age, sex, HR, PP, SBP, DBP, MBP, HbAlc percentage, and levels of hsCRP, total cholesterol, HDL, and LDL (Table 1).

Among the study patients, 84\% (54/64) had a history of hypertension and $31 \%(20 / 64)$ had diabetes mellitus. The proportion of subjects with hypertension was similar in the deep and lobar ICH groups (39/45 vs. $15 / 19$, respectively; $P=0.46$, Fisher test). No differences were found regarding the proportion of patients with diabetes mellitus in the 2 groups (16/45 vs. $4 / 19$, respectively; $P=0.37$, Fisher test). In the patients with deep ICH, ASI was significantly higher than in the patients with lobar ICH (0.64 \pm 0.19 vs. $0.53 \pm 0.17, P=0.04)$.

\section{Discussion}

The main result of our study is the higher ASI values in deep hemorrhages than in lobar hemorrhages. The predominant lo- cation of ICH within the brain varies in different populations. In particular, in the United States and Europe, deep ICH is more common than lobar $\mathrm{ICH} .{ }^{13}$ According to data obtained from the literature and our study design, which included an enrollment of consecutive primary ICH patients, we observed a difference in sample size between the deep and lobar ICH groups. Furthermore, the number of patients in the lobar ICH group was reduced by an additional number of patients with a secondary cause of hemorrhage. The evidence of different patterns of these two subtypes in race-ethnically diverse population suggests the notion that $\mathrm{ICH}$ should no longer be treated as a single entity; ${ }^{13}$ these epidemiological differences between the deep and lobar subtypes confirm our hypothesis that $\mathrm{ICH}$ is a heterogeneous disease, with probable different pathogenetic mechanisms.

Arterial stiffening plays a key role in the pathophysiological mechanism of the cardiovascular system, predicting the incidence of cardiovascular complications, ${ }^{7}$ with a prognostic value in patients with spontaneous $\mathrm{ICH} .{ }^{14}$ A previous study showed higher ASI values in acute ischemic stroke, particularly in the lacunar subtype, ${ }^{9}$ suggesting a strict association between hypertension and diabetes in the lacunar group. According to the hypothesis that a similar pathogenesis may underlie hypertensive $\mathrm{ICH}$ and lacunar disease, we showed in our study that ASI was higher in the deep ICH patients than in the lobar ICH patients.

Vascular stiffening develops from a complex interaction between stable and dynamic changes involving structural and cellular elements of the vessel wall. Arterial stiffness represents the result of molecular, cellular, and genetic factors, which can be a consequence of many disease states such as hypertension, diabetes, atherosclerosis, hypercholesterolemia, and chronic renal compromise. ${ }^{10}$ In our study, because of the small sample size, we could not perform a subgroup analysis according to the dif- 
ferent risk factors (hypertension, hypercholesterolemia, inflammation, and diabetes); for this reason, all other metabolic parameters and BP profiles were not significantly different between the two groups. However, the results showed higher ASI values in the deep ICH patients, suggesting a stiffer arterial tree in the patient group, regardless of the underlying risk factors.

Elasticity of the great vessels is an important function of the vascular system. Owing to this property, large changes in $\mathrm{BP}$ and blood flow that result from intermittent ventricular ejection are integrated into a more steady flow within peripheral tissues. ${ }^{15}$ The aortic wall, which contains a high proportion of elastin fibers, permits a significant distension during systole; whereas during diastole, blood is pushed forward through the arterial tree due to elastic recoil, ensuring blood flow in one direction and its stabilization in peripheral tissues. The more-distal muscular arteries differ from the aorta in that they have a higher proportion of collagen fibers, making them less distensible. The net result is a progressive reduction of pulsatility through the arterial tree to the level of the microcirculation of high-flow cerebral and renal vascular beds. This minimizes the barotrauma that would result from exposure to peak systolic pressures.

As blood vessels become stiffer, the pulse wave is transmitted more rapidly and returns to the heart during left ventricular contraction, resulting in a greater augmentation of the central aortic systolic pressure. Stiffened arteries require a greater amount of force to expand and take up the blood ejected from the heart. This increased force requirement is provided by the heart, which must contract more forcefully to accommodate the arteries. The vasculature of the brain is exposed to greater pressure fluctuations that are linked to an increased risk of stroke. In fact, most of the bleeding in deep ICH occurs at or near the bifurcation of small penetrating arteries that originate from basilar arteries or the anterior, middle, or posterior cerebral arteries. Small artery branches often have multiple sites of rupture; some are associated with layers of platelet and fibrin aggregates. These lesions are characterized by breakage of elastic lamina, atrophy and smooth muscle fragmentation, dissections, and granular or vesicular cellular degeneration. Fibrinoid necrosis of the subendothelium with subsequent focal dilatations (micro aneurysms) leads to vessel rupture in a small proportion of patients.

Finally, stiffened arteries, which require a greater amount of force to expand, expose the vasculature of the brain to greater pressure fluctuations that are linked to an increased risk of hemorrhage and stroke. The hypothesis that arterial stiffness may play a key role in the pathophysiological mechanism of deep cerebral hemorrhage is suggested also by a study that indicated an association between arterial stiffening and the presence of microbleeds. ${ }^{16}$ Furthermore, a recent study ${ }^{17}$ showed that arterial stiffening was independently associated with nonlobar cerebral microbleeds, but not with lobar cerebral microbleeds, confirming a different pathogenesis in the two groups.

Moreover, a previous study suggested that arterial stiffening in patients with spontaneous ICH was a predictor of mortality at 6 months. ${ }^{14}$ In this view, drug treatment could prevent deep hemorrhagic stroke through a reduction in arterial stiffness in parallel with the correction of cardiovascular risk factors such as hypertension, dyslipidemia, diabetes mellitus, and smoking, which are all associated with arterial stiffening. ${ }^{18}$ Promising therapeutic strategies to reduce arterial stiffness include taking advantage of the non-lipid-lowering effects of statins and directly targeting the molecular events that lead to arterial stiffening, such as formation of advanced glycation end products.

In fact, patients with hypercholesterolemia are well known to have stiffer blood vessels than control subjects, despite similar BPs and mechanisms linking arterial stiffening to plasma lipids, including atherosclerosis, changes in the elastic elements of the arterial wall, and endothelial dysfunction. ${ }^{19}$ Statins and other cholesterol-reducing agents have been shown to have beneficial effects on wave reflection and aortic stiffening in several patient groups. ${ }^{20,21}$ Furthermore, the important role of inflammation has become apparent in the stiffening of large arteries, possibly via changes in the composition of the arterial wall due to inflammatory cell infiltration or via endothelial dysfunction. Recent studies in healthy individuals and in patients with isolated systolic hypertension indicated an association between arterial stiffening and serum CRP levels, ${ }^{22}$ suggesting the inflammation as a potential target for reduced arterial stiffness. In diabetes, advanced glycation end products accumulate in extracellular matrix proteins such as collagen and contribute to vessel stiffening. ${ }^{23,24}$ Prevention of glycation reactions may be a target for reducing the deterioration of vascular elastic properties.

One of the limitations of the present study is the relatively small number of patients. A larger number of patients is necessary to generalize the results of this study. In addition, the sample size of the patient group with lobar hemorrhage was smaller than that of the patient group with deep hemorrhage. The lower incidence of lobar hemorrhage in the patients who referred consecutively to our department for ICH explains this difference in sample size between the two groups.

\section{Conclusion}

This study suggests that, in deep ICH, arterial stiffness represents a possible pathogenetic factor able to modify arterial wall properties so contributing to vascular rupture in response to acute blood pressure elevation: therapeutic strategies that re- 
duce arterial stiffness may potentially lower the incidence of deep hemorrhagic stroke.

\section{References}

1. van Asch CJ, Luitse MJ, Rinkel GJ, van der Tweel I, Algra A, Klijn CJ. Incidence, case fatality, and functional outcome of intracerebral haemorrhage over time, according to age, sex, and ethnic origin: a systematic review and meta-analysis. Lancet Neurol 2010;9:167-176.

2. Qureshi $\mathrm{AI}$, Mendelow $\mathrm{AD}$, Hanley DF. Intracerebral haemorrhage. Lancet 2009;373:1632-1644.

3. Sutherland GR, Auer RN. Primary intracerebral haemorrhage. J Clin Neurosci 2006; 13:511-517.

4. Labovitz DL, Halim A, Boden-Albala B, Hauser WA, Sacco $\mathrm{RL}$. The incidence of deep and lobar intracerebral hemorrhage in whites, blacks, and hispanics. Neurology 2005;65:518-522.

5. Labovitza DL, Sacco RL. Intracerebral hemorrhage: update. Curr Opin Neurol 2001;14:103-108.

6. Luft FC. Molecular mechanisms of arterial stiffness: new insights. J Am Soc Hypertens 2012;6:436-438.

7. Adji A, O’Rourke MF, Namasivayam M. Arterial stiffness, its assessment, prognostic value, and implications for treatment. Am J Hypertens 2011;24:5-17.

8. Hatanaka R, Obara T, Watabe D, Ishikawa T, Kondo T, Ishikura K, et al. Association of arterial stiffness with silent cerebrovascular lesions: the Ohasama study. Cerebrovasc Dis 2011;31: 329-337.

9. Tuttolomondo A, Di Sciacca R, Di Raimondo D, Serio A, D’Aguanno G, Pinto A, et al. Arterial stiffness indexes in acute ischemic stroke: relationship with stroke subtype. Atherosclerosis 2010;211:187-194.

10. Zieman SJ, Melenovsky V, Kass DA. Mechanisms, pathophysiology, and therapy of arterial stiffness. Arterioscler Thromb Vasc Biol 2005;25:932-943.

11. Li Y, Wang JG, Dolan E, Gao PJ, Guo HF, Nawrot T, et al. Ambulatory arterial stiffness index derived from 24-hour ambulatory blood pressure monitoring. Hypertension 2006;47:359-364.

12. Dolan E, Thijs L, Li Y, Atkins N, McCormack P, McClory S, et al. Ambulatory arterial stiffness index as a predictor of cardio- vascular mortality in the Dublin Outcome Study. Hypertension 2006;47:365-370.

13. Flaherty ML, Woo D, Haverbusch M, Sekar P, Khoury J, Sauerbeck $\mathrm{L}$, et al. Racial variations in location and risk of intracerebral hemorrhage. Stroke 2005;36:934-937.

14. Keong LH, Ghani AR, Awang MS, Sayuthi S, Idris B, Abdullah $\mathrm{JM}$. The role of a high augmentation index in spontaneous intracerebral hemorrhage to prognosticate mortality. Acta Neurochir Suppl 2011;111:375-379.

15. Quinn U, Tomlinson LA, Cockcroft JR. Arterial stiffness. JRSM Cardiovasc Dis 2012;1:cvd.2012.012024.

16. Ochi N, Tabara Y, Igase M, Nagai T, Kido T, Miki T, et al. Silent cerebral microbleeds associated with arterial stiffness in an apparently healthy subject. Hypertens Res 2009;32:255-260.

17. Song TJ, Kim J, Kim YD, Nam HS, Lee HS, Nam CM, et al. The distribution of cerebral microbleeds determines their association with arterial stiffness in non-cardioembolic acute stroke patients. Eur J Neurol 2014;21:463-469.

18. Laurent S, Boutouyrie P. Arterial stiffness and stroke in hypertension: therapeutic implications for stroke prevention. CNS Drugs 2005; 19:1-11.

19. Wilkinson I, Cockcroft JR. Cholesterol, lipids and arterial stiffness. Adv Cardiol 2007;44:261-277.

20. Mäki-Petäjä KM, Wilkinson IB. Anti-inflammatory drugs and statins for arterial stiffness reduction. Curr Pharm Des 2009; 15: 290-303.

21. Wang ZG, Chen BW, Lü NQ, Cheng YM, Dang AM. Relationships between use of statins and arterial stiffness in normotensive and hypertensive patients with coronary artery disease. Chin Med J (Engl) 2013;126:3087-3092.

22. Mäki-Petäjä KM, Wilkinson IB. Arterial stiffness and inflammation: a potential target for a drug therapy. Artery Res 2010;4:99107.

23. Llauradó G, Ceperuelo-Mallafré V, Vilardell C, Simó R, Gil P, Cano A, et al. Advanced glycation end products are associated with arterial stiffness in type 1 diabetes. J Endocrinol 2014;221: 405-413.

24. Stephen EA, Venkatasubramaniam A, Good TA, Topoleski LD. The effect of glycation on arterial microstructure and mechanical response. J Biomed Mater Res A 2014;102:2565-2572. 